\title{
Osteopenia de niños nacidos antes de término. Evaluación mediante densitometría ósea
}

\author{
John Wilson Sch. ${ }^{1}$; Andrés Román N.'; Juan Pablo Beca 1.1; Jose M. Cortés E. ${ }^{1}$; \\ Horacio Amaral P.2; Bárbara Morales K.2; Ana María Espíndola A. ${ }^{3}$
}

\section{Bone densitometry in osteopenia of prematurity}

\begin{abstract}
Osleopenia of prematury is seen more hrequently as more very low birthweight babies survive. Dlagnosis based on classicol radiologicol or alcaline phostatase delerminations is not reliable. Bone densitometry hos rarely been used at this age. In order lo asses its volue in the diognosis of osteopenia of premalurity, 12 preterm babies lgestational age 25 to 30 weeks 1 and 10 control babies (36 and 37 weeks geslational age) were studied prospectively. Deleıminations were done in a double photon 2600 Norlan densitometer. Volues were expresed as TBM (total bone mineral), TBMN Weight, and $\mathrm{BMD}$ (bone mineral densilyl. Contials had a mean birth weight of $2833 \mathrm{~g}$, all were exclusively breast fed and were sludied before age 3 days. Mean birth weight of study group was $1238 \mathrm{~g}$ they were studied at 30 and 44 weeks postconceptional age and they were fed wilh their own's mother milk or a formula which contains $80 \mathrm{mg} \% \mathrm{Ca}$ and $40 \mathrm{mg} \% \mathrm{P}$. Control group bobies had TBM $54.9 \pm 0.05 \mathrm{~g}$. TBM/Weight $19.65 \pm 1.98 \mathrm{~g}$. and BMD $0.531 \pm 0.06 \mathrm{i}$ $\mathrm{g} / \mathrm{cm}^{2}$. Newborn bobies $\leq 30$ weeks of 36 weeks correcled age had significantly lower values: TBM $27.75 \pm 0.0 \mathrm{~g}$. TBM/Weight $10.80 \pm 1.0 \mathrm{~g}$ and $\mathrm{BMD} 0.202 \pm 0.05 \mathrm{~g} / \mathrm{cm}^{2}$. At 44 weeks this values rose wo TBM $54.98 \pm 14.5 \mathrm{~g}$ [ns), TBM, Weight $12.0 \pm 2.22 \mathrm{~g}$ ip $<0.0001$ and $B M D 0.346 \pm 0.00 \mathrm{~g} / \mathrm{cm}^{2}$ ip $<0.0001$. TBM/Weight and $B M D$ are the most important porameters and both were still significantly lower in preterm Infonts than in controls at age 44 weeks. Eicne densilomeriy is a usehul melhod to sludy bone mineralizalion in premature babies and its change with oge. It can also be useful to monitor freatment and nutrition.
\end{abstract}

[Key words: osleopenia, preterm infants, premalurity, bone densilemetry.]

En la medida en que sobreviven cada día más recién nacidos de muy bajo peso, aparecen con mayor frecuencia problemas de desmineralización ósea, clínicamente conocida como osteopenia del pretérmino. La afección puede tener distinta intensidad, llegando en casos extremos a causar fracturas patológicas. En aquellos recién nacidos (RN) que, además tienen afecciones propias de su inmadurez como es el caso de la displasia broncopulmonar, Ia osteopenia puede ser muy severa, sobre todo si se usan diuréticos por largo tiempo en el manejo.

En la práctica clínica se emplean las fosfatasas alcalinas y la radiología convencional como una forma objetiva de medir el grado de mineraliza-

1. Servicio de Recién Nacido de Clínica Alemana.

2. Servicio de Medicina Nuclear de Clínica Alemana.

3. Enfermera Universilaria. Servicio de Recién Nacido de Clínica Alemana. ción ósea de estos pacientes. Se determina asi la alimentación más adecuada y los suplementos de calcio y fósforo que puedan ser necesarios ${ }^{1,2}$. La densitometría ósea (DO) es un método desarrollado en los últimos años, que permile deterninar el contenido mineral óseo con bastante exactitud. Si bien existen publicaciones de tipo experimental, que analizan el contenido mineral óseo de RN de bajo peso, la mayoría de los estudios de $D O$ se refieren a adultos, en quienes hay una experiencia importante. La mayoria de publicaciones sobre $\mathrm{RN}$ se refieren a trabajos que se efectuaron con densitómetros de un fotón. Esa técnica no es de gran precistón, ya que la medición se hace en el brazo del $\mathrm{RN}_{\text {, }}$ en cambio la de doble fotón, desarrollada posteriormente, mide el contenido mineral de cuerpo entero y con bastante rapidez lo que, sin duda, le da mayor exactitud $d^{3-11}$.

Con el objetivo de conocer el aporte de la densitometría ósea en el diagnóstico de la osteopenia del prematuro se estudió en forma 
prospectiva un grupo de recién nacidos de 300 menos semanas de gestación.

\section{Material y Método}

Se estudiaton 12 recién nacidos pretérmino, de 30 o menos semanas de gestación, sin excluir a ninguno por sus enfermedades concomitantes. Además se esiudió un grupo control de 10 recién nacidos sanos de 36 a 37 semanas de gestación. La densitometría ósea se efecluó con un densitómetro Norland modelo 2600 de doble fotón. La densitometría osea de doble fotón permite medir el contenido mineral de cuerpo entero. La tếcnica utiliza un emisor de dos rayos gamma que hace un barrido sobre la zona en estudio. La diferencia de atenuación entre ambos rayos Bamma es analizada por un sistema computacional, lo que pemite distinguir entre tejido óseo y blando. Asimismo, mediante una ccuaciós, se puede calcular la masa mineral por área $(g / \mathrm{cm} 2)$, unidad en que, con esta lécnica se expresa Ia densidad ósea. Como se conoce el área ósea, tambión puede calcularse el contenido mineral óseo total. I.as mediciones se hicieron de cuerpo entero, e incluyeron TBM (contenido mineral óseo total), TBM/peso y BMD (densidad mineral total). En todos los casos se solicitó el cunsentimiento por escrito a los padres, previa información detallada de los exámenes que a cada niño se le practicariat.

En el grupo control se incluyeron 10 niños sanos, de gestación de duración conocida (36 a 37 semanas). calculando que ésta fuese comparable con la que tendrían los niños del grupo estudio al momento de realizar, en estos últimos, la primera medición. Su peso promedio cra $2833 \mathrm{~g}$ (2 500 a $3560 \mathrm{~g}$ ). Su alimentación se hizo exclusivamente por pecho. Las mediciones se hicieron antes de los tres días de vida.

En el de grupo estudio se inchyeron $12 \mathrm{RN}$ de pretérmino con gestación promedio de 28,5 (25 a 30) semanas y peso promedio al naces de $1230 \mathrm{~g}(873$ a 1585$)$. Estos RN presentaron diversas afecciones relacionadas con su prematurez, sir embargo ninguno sufrió displasia broncopulmonar y los diuréticos se usaron sólo ocssionalmente en algunos casos y siempre en dosis aisladas. Todos recibieron alimentación con leche materna of 6 mula con $80 \mathrm{mg} \%$ de calcio y $40 \mathrm{mg} \%$ de fósforo. En cada uno de ellos se rcalizaron dos mediciones: en la primera, a las 36 semanas de edad postconcepcional, el peso promedio era de $2534 \mathrm{~g}$ (1 640 a $3046 \mathrm{~g}$ ). En la segunda a las 44 semanas, el peso promedio era $4541 \mathrm{~g}$ (2929 a $5797 \mathrm{~g}$ ).

El análisis de significado estadístico de los resultados se hizo con la pruebe $z$ de Student.

\section{Resultados}

Las densitometrías óseas del gtupo control mostraron un contenido mineral óseo total (TBM) de $54,9 \pm 9,05 \mathrm{~g}$, TBM/peso $19,65 \pm 1,98 \mathrm{~g} \mathrm{y}$ densidad ósea de $0,531 \pm 0,061 \mathrm{~g} / \mathrm{cm}^{2}$ (tabla 1 ). En el grupo de estudio, la primera determinación mostró TBM 27,7 $\pm 6,6 \mathrm{~g}$. TBM/peso $10,86 \pm 1,6$

\section{rabla 1}

Resultados de la densitometría ósea en recién nacidos sanos de 36 a 37 semanas de edad gestaciona!

\begin{tabular}{ccccc}
\hline Caso & Peso (g) & TBM (g) & TBM/peso & BMD (g/cm) \\
\hline 1 & 3560 & 56,1 & 17,10 & 0,536 \\
2 & 3147 & 64,3 & 21,72 & 0,592 \\
3 & 2500 & 48,7 & 19,87 & 0,505 \\
4 & 2623 & 45,6 & 16,70 & 0,507 \\
5 & 2529 & 55,5 & 21,51 & 0,540 \\
6 & 3043 & 70,2 & 22.21 & 0,549 \\
7 & 3089 & 64,5 & 21,35 & 0,649 \\
8 & 2948 & 53,5 & 18,83 & 0,510 \\
9 & 2543 & 43,1 & 18,34 & 0,513 \\
10 & 2618 & 47,5 & 18,92 & 0,414 \\
\hline $\bar{x}$ & 2833 & 54,9 & 19,65 & 0,531 \\
\hline D E & \multicolumn{5}{c}{} & 9,05 & 1,98 & 0,061 \\
\hline
\end{tabular}

g y densidad osea de $0,262 \pm 0,05 \mathrm{~g} / \mathrm{cm}^{2}$. En la segunda decerminación realizada a las 44 semanas de gestación, el contenido mineral óseo aumentơ a $54,9 \pm 14,5 \mathrm{~g}$, el TBM/peso a $12,0 \pm 2,22 \mathrm{~g}$, y la densidad ósea a $0,346 \pm 0,06 \mathrm{~g} / \mathrm{cm}^{2}$ en Jos niños pretérmino (tabla 2).

El análisis del contenido mineral óseo total (figura 1) mostró diferencia significativa $(p<$ 0,0001 ) entre los niños control y pretérmino a las 36 semanas y entre la primera y segunda determinación del grupo de estudio. Las determinaciones de TBM/peso mostraron diferencia significativa ( $p<0,0001)$ entre controles y la primera deterninación en los recién nacidos pretérmino. Entre la primera y la segunda determinación no hubo, sin embargo, cambios significativos en TBM/peso (figura 2). La densidad ósea fue significativamente diferente entre la primera determinación de los recién nacidos pretérmino y controles ( $\mathrm{p}<0,0001)$ y no hubo diferencia significativa entre ambas determinaciones del grupo dc estudio (figura 3 ).

\section{Comentario}

Se eligieron, para estc estudio, recién nacidos de 30 o menos semanas de gestación por su alto riesgo de sufrir desmineralización ósea, aunque no presenten signos de raquitismo clínico o ra- 


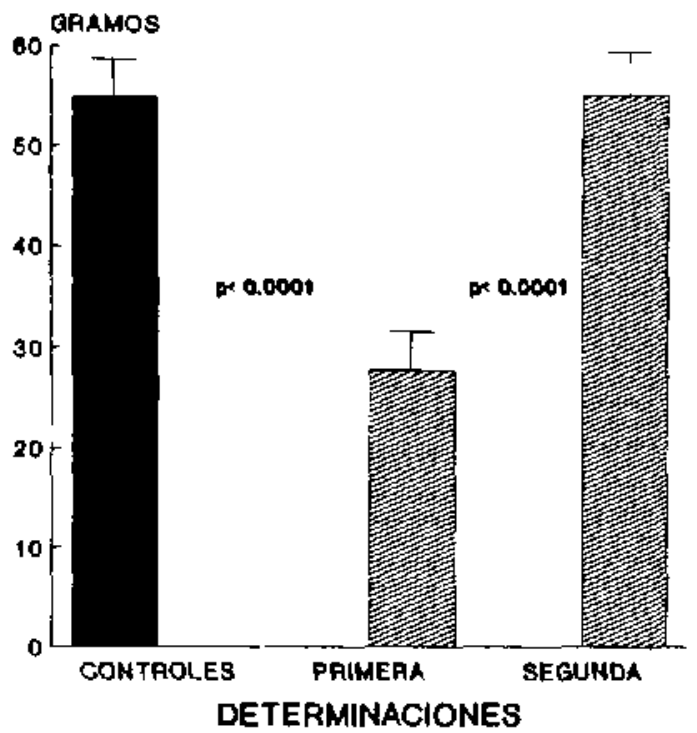

CONTROLES GRUPO ESTUDIO

Higura 1: Contenido mineral óseo total (TBM). Existen diferencias significativas cntre las delerminaciones del grupo contool y la primera dcteminación del grupo de escudio y también entre la primera y segunda deterninación de csle grupo.

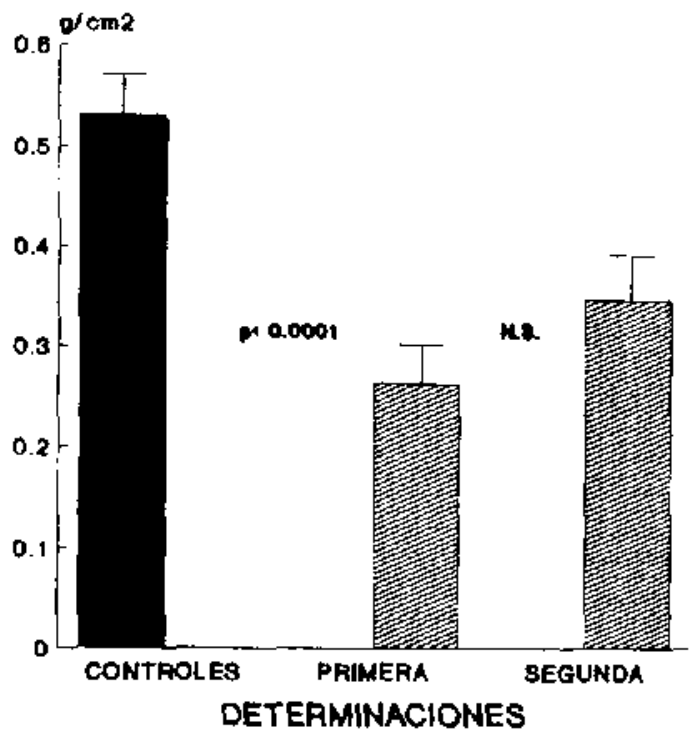

CONTHOLES GROPO ESTUDIO

Figura 3: Densidad mineral ósea (BMD). La densidad bsea fue significalivamente diferente entre cl grupo de control y la primera deteminación del grıpo de estudio.

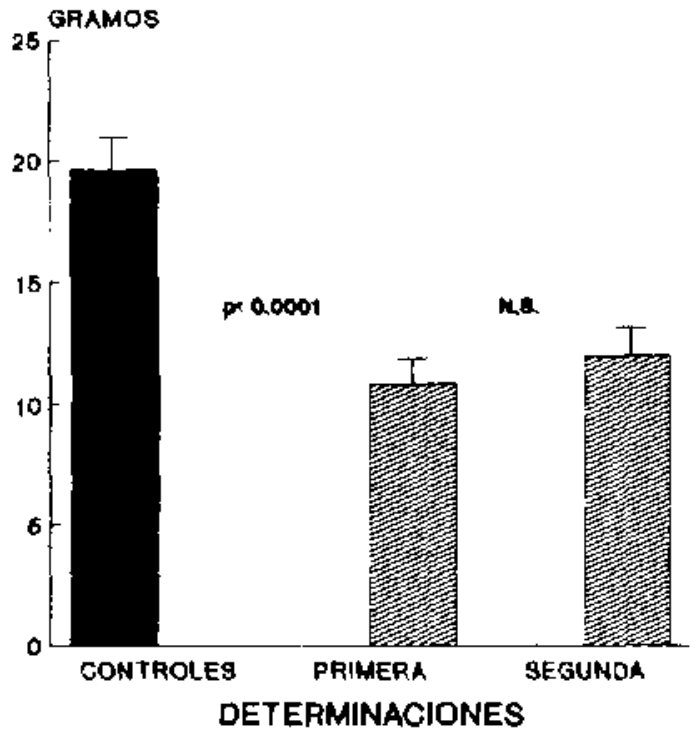

CONTAOLES GRUPO ESTUDIO

Figura 2: TBM/peso. Existen difcrencias significativas entre el grupo de control y la primera dcteminación del grupo de estudio. No existen diferencias entre la primera y scgunda determinación del grupo de cstudio.

diológico. Ninguno de los niños incluidos tuvo esta complicación ni afecciones como displasia broncopulmonar o uso prolongado de diuréticos que aumentan el riesgo de osteopenia. La cdad de la primera medición del estudio se decidió en base a su factibilidad, ya que la desitometría ósea se hace sobre una camitla que no es posible caleCaccionar en forma adecuada, con riesgo de enfriamiento en los recién nacidos menores. La segunda densitometría se hizo a las 44 semanas postconcepcionales permitiendo que iranscurriese suficiente tiempo para observar posibles modificaciones en cl contenido mineral ósco, para lo cual los pacientes fueron estudiados ambulatoriamentc.

Como grupo control se cscogieron recien nacidos de 36 a 37 semanas de gestación para comparar sus resultados con los primeros del grupo de estudio a las 36 semanas postconcepcionales. Podria ser discutible hacer comparaciones de recién nacidos de pretérmino, con 30 o menos semanas đe gestación y alimentados por vía oral por varias semanas, con recién nacidos de 36 a 37 semanas y sólo 3 días de vida. A pesar de esta 
Tabla 2

Resultados de la densitometría b́sea en recién nacidus pretérmino, edad gestacional de 25 a 30 semanas

\begin{tabular}{|c|c|c|c|c|c|c|}
\hline \multirow{2}{*}{$\begin{array}{l}\text { Caso } \\
\text { Determinación }\end{array}$} & \multicolumn{2}{|c|}{ TBM (g) } & \multicolumn{2}{|c|}{ THM/peso } & \multicolumn{2}{|c|}{ ПNID $(\mathrm{g} / \mathrm{cm})$} \\
\hline & $1 !$ & $2^{2}$ & $1^{1}$ & $2 !$ & $1^{3}$ & $2 !$ \\
\hline 1 & 25.6 & 59,6 & 9,79 & 13,18 & 0,245 & 0,382 \\
\hline 2 & 17,7 & 28,6 & 8,87 & 8.09 & 0,258 & 0,334 \\
\hline 3 & 37,2 & 57,3 & 12,21 & 11,98 & 0,383 & 0,365 \\
\hline 4 & 32,1 & 63,3 & 12,21 & 12,28 & 0,265 & 0,371 \\
\hline 5 & 30,1 & 65,0 & 10,43 & 13,25 & 0.219 & 0,395 \\
\hline 6 & 29,1 & 46.1 & 11.68 & 10,73 & 0,252 & 0,314 \\
\hline 7 & 18,3 & 35,3 & 11.15 & 12.05 & 0,232 & 0,268 \\
\hline 8 & 18,1 & 38,0 & 8,11 & 8.55 & 0.182 & 0.196 \\
\hline 9 & 37,7 & 82,5 & 14,25 & 16,76 & 0,272 & 0,417 \\
\hline 10 & 32,4 & 64,9 & 11,38 & 11,19 & 0,322 & 0,372 \\
\hline 11 & 26,2 & 60,4 & 9,95 & 14,00 & 0.281 & 0,357 \\
\hline 12 & 28,5 & 58,8 & 10.35 & 12,00 & 0,244 & 0.386 \\
\hline$\overline{\mathrm{x}}$ & 27,7 & 54,9 & 10,86 & 12,00 & 0,262 & 0,346 \\
\hline$D E$ & 6,6 & 14,5 & 1,6 & 2,22 & 0,05 & 0,06 \\
\hline
\end{tabular}

limitación se definió el grupo control del modo indicado, considerando que ellos aportan valores de contenido mineral normales para la edad y podrían considerarse como meta a alcanzar con una nutricion ideal.

Los resultados demostraron que en los recién nacidos de pretérmino el contenido mineral oseo es muy inferior al de los controles a la misma edad postconcepcional, to que es concordante con 10 informado por otros $4,9,30$ y significa desmineralización ósea subclínica. El aporte de calcio en la dieta fue de $128 \mathrm{mg}-\mathrm{kg}$ de peso-día. Los contenidos minerales más bajos se observaron en dos niños que recibieron sólo leche materna, lo cual confirma la necesidad de suplementar con calcio la nutrición de los rocién nacidos de pretćrmino alimentados en forma natural. Ninguno de los pacientes estudiados tuvo signos clínicos o radiológicos de raquilismo. Es posible que casos con raquitismo clinico o enfermedades agravantes de la osteopenia del pretérmino presentasen valores de densitometría ósea inferiores a los de esta scric.

En la determinación de contenido mineral a las 44 semanas de edad postconcepcional se observó aumento importante del contenido mineral total, que alcanzó valores jguales a los del grupo control. Sin embargo, el contenido mineral por $\mathrm{kg}$ de peso (TBM/peso) y la densidad ósea (g/ $\mathrm{cm}^{2}$ ) no se comigieron, lo que es atribuible af gran incremento del peso y la talla obscrvado entre ambas determinaciones. En efecto, el peso promedio enure ambos períodos aumentó de 2534 a $4541 \mathrm{~g}$. Sc pucde suponer, dado el aumento del contenido mineral total, que los valores de TBM/ peso y BMD tienden a mejorar con el tiempo, lo cual debería demostrarse con estudios de seguimiento a mayor plazo.

Del presente estudio se puede concluir que los $\mathrm{RN}$ de 30 o menos scmanas de gestación presentan menor mineralización ósea a pesar de un buen incremento ponderal y que su recuperación en el tiempo cs lenta. De las mediciones efectuadas debe destacarse que el contenido mineral óseo total (TBM) es un parámetro de poca significación si no se calcula el TBM/peso o el contenido mineral por superficie o BMD. Las delerminaciones de densitometría ósea con densitómetro de doble fotón constituyen un valioso aporte en el seguimiento nutricional y en cstudios de formulas especiales para la alimentación de recién nacidos de pretérmino. 


\section{Resumen}

La osteopenia del pretérmino es cada vez más frecuente. Los métodos que actualmente se ernplean para evaluarla son las fosfatasas alcalinas y la radiología, de poca precisión. La densitometría ósea es más precisa, pero hay poca experiencia con ella en el período neonatal. Con el objetivo de describir su aporte, en el diagnóstico de la osteopenia del prematuro, se estudiaron 12 RN pretérmino de 25 a 30 semanas de gestación; y $10 \mathrm{RN}$ sanos de 36 a 37 semanas de edad gestacional como controles. La medición se efecluó con densitómetro Norland 2600 de doble foton. Se establecieron valores para TBM (contenido mineral óseo total), TBM/peso y BMD (densidad mineral ósea). El grupo control tuvo un peso promedio de $2833 \mathrm{~g}$ y la medición se efectuó en los primeros tres días de vida. En el grupo de estudio, la edad gestacional promedio fue 28,5 semanas, el peso al nacer $1238 \mathrm{~g}$, las mediciones se efectuaron a las 36 y 44 scmanas postconcepcionales y su alimentación se hizo con leche materna o fórmula con $80 \mathrm{mg} \%$ de Ca y $40 \mathrm{mg} \%$ de $\mathrm{P} O$ ambas. Los RN controles tuvieron TBM $54,9 \pm 9,05 \mathrm{~g}, \mathrm{TBM} /$ peso $19,65 \pm 1,98$, y BMD $0,531 \pm 0,0061 \mathrm{~g} / \mathrm{cm}^{2}$ a la edad de tres días. En los RN pretérmino, a las 36 semanas los resultados fueron: TBM $27,75 \pm 6,6 \mathrm{~g}, \mathrm{TBM}$ /peso 10,86 $\pm 1,6$ y BMD $0,262 \pm 0,05 \mathrm{~g} / \mathrm{cm}^{2} y$, a las $44 \mathrm{sc}-$ manas, TBM $54,98 \pm 14.5 \mathrm{~g}$ (ns), TBM/peso $12,0 \pm 2,22(\mathrm{p}<0,0001), y$ BMD $0,346 \pm 0,06 \mathrm{~g})$ $\mathrm{cm}^{2}$ (p <0,0001). TBM/peso y BMD, a las 44 semanas persistían significativamente más bajos que el grupo control. La densitometría ósea es úlil para demostrar la disminución del contenido mineral óseo en prematuros, seguir su evolución y controlar su manejo.
(Palabras clave: recićn nacido, prematuro, osteopenia, densitometría ósea.)

\section{Agradecimientos}

Los autores agradecen la colaboración del personal de enfermería del Servicio de Nconatología de la Clínica Alemana y la asesoría cstadística de la Srta. Gloria Icaza.

\section{Referencias}

1. Forbes $G$. Some remarks on bone minctalization. J Pediatr 1988: 113: 167-171.

2. Minson $S$, Steichen $J$ and Tsang $R$ : Decreased Bonc Mineral Content in Small-for-Gestational-Age Infants Compared with Appropiate-for-Gestational Infants: Normal Serum 25-Hidroxyvitamin D and Decreasing Parathyroid Homone. Pedistrics 1983; 71 : 383-388.

3. Minton S, Steichen f and Tsang R: Bone Mincral Content in Tenn and Preterm Appropriate-for-Gestational-Age Infants. J Pedials 1979; 95: 1037-1042.

4. Barden $H$ and Mazess $R$ : Bone densitometry in infants J Pediatr 1988; 113: 172-177.

5. Mimouni $F$ and Tsang $R$ : Bone mineral content: Data analysis. J Pediats 1988; 113: 178-180.

6. Steichen $J$. Steichen $P$ and Tsang $R$ : Bone mincral content measurement in small infants by single-photon absorptiometry: Current metodologic issues. J Pediatr 1988; 113:181-187.

7. Vyhmeister $N$ and Linkhari T: Mcasurement of hu. menus and radius bone minetal content in the pretcm and tern infant. J Pediatr 1988; 113: 188-195.

8. Petersen S, Gotfredsen $A$ and Krudsen $F$ : Lean body mass in small for gestational age and appropriate for gestational age infants. J Pedriatr 1988; 113: 886-889.

9. Petersen S, Gotfredsen A and Knudsen F: TeLal Body Bone Mineral in Light-for-Gestational-Age Infants and Appropriate-for-Gcstational-Age Infants. Acta Paediats Scand 1989: $78: 347$.

10. Mazess R: Bone densitometry in infants. J Pedialr 1988; 113: 196-204.

11. Horsman A, Ryan SW, Congdon PJ, Truscott JG, Sim. psan $M$ : Bonc mineral content and body size Prelem and full term infants. Arch Dis Child 1989; 64: 1579-1586. 